\title{
ON THE NONLINEAR ANALYSIS OF OPTICAL FLOW
}

\author{
ShengXiang Xia - Yanmin Yin
}

\begin{abstract}
We utilize the methods of computational topology to the database of optical flow created by Roth and Black from range images, and demonstrate a qualitative topological analysis of spaces of $3 \times 3,5 \times 5$ and $7 \times 7$ optical flow patches. We experimentally prove that there exist subspaces of the spaces of the three sizes high-contrast patches that are topologically equivalent to a circle and a three circles model, respectively. The Klein bottle is the quotient space described as the square $[0,1] \times[0,1]$ with sides identified by the relations $(0, y) \sim(1, y)$ for $y \in[0,1]$ and $(x, 0) \sim(1-x, 1)$ for $x \in[0,1]$. For the space of $3 \times 3$ optical flow patches we found a subspace having the same homology as that of the Klein bottle. As the size of patches increases, the Klein bottle feature of the spaces of $5 \times 5$ and $7 \times 7$ optical flow patches gradually disappears.
\end{abstract}

\section{Introduction}

A pixel of an image taken by a digital camera has a gray scale value. Thus, each range image can be considered as a vector in a very high-dimensional space $X$. What can be said about the set of images $M \subseteq X$ which one gets when one takes many images by a digital camera? The direct study of $M$ is very hard, because $M$ is very high-dimensional and very sparse in $X$. An approximation is to analyze the space of local patterns of pixel values, modeled by small patches of images. There are many advantages in analyzing a space of images

2010 Mathematics Subject Classification. 62H35, 65D18.

Key words and phrases. Optical flow; persistent homology; high-contrast patches; Klein bottle.

The project is supported by the National Natural Science Foundation of China (Grant No. 61471409). 
locally. Firstly, this greatly reduces the dimensional problem. Next, it has been observed by Field ([10]) and van Hateren ([18]) that understanding of the local statistics provides a lot of information about the global statistical properties of the image. In the paper [15], Lee, Pedersen, and Mumford study the distributions of $3 \times 3$ patches from optical and range images, they have a number of very interesting observations about the resulting space of patches, for example, they find that the majority of the high-contrast $3 \times 3$ optical patches are concentrated near a 2-dimensional annulus. In the paper [7], Carlsson, Ishkanov, de Silva, and Zomorodian apply computational topological tools to the dataset of optical patches studied by Lee, Pedersen, and Mumford [15], they find that there exists one high-density subset called the primary circle, and prove that there exists a large 2-dimensional subset with the topology of a Klein bottle that contains the primary circle, which could improve the technique of image compression ([7]). In the paper [2], Adams and Carlsson found that $5 \times 5$ and $7 \times 7$ range patches have the primary circle behavior.

The concept of optical flow was introduced by James J. Gibson in the 1940s to represent the visual stimulus supplied to animals moving through the world [13]. Optical flow is the apparent motion of objects in a visual scene originated by the relative motion between the viewer and the scene [19]. Optical flow has many applications such as object segmentation, tracking, motion estimation and video compression. Optical flow estimation is one of the basic research topics in computer vision, there are plenty results on the topic achieved in recent years [4], [5], [14], [17]. Natural image statistics has obtained in-depth study, due to the difficulty of obtaining ground truth data for modeling optical flow statistics, while the spatial statistics of optical flow is relatively undeveloped. In the paper [16], Roth and Black constructed a database of natural scene motions by making use of range images and camera motions (some other databases of optical flow are available in [4], [11]), they studied the spatial statistics of optical flow and gained a rich prior model of optical flow. In the paper [1], Adams, Atanasov, and Carlsson applied the nudged elastic band technique to analyse data of the database, they found a new topological feature of an optical flow data set for $3 \times 3$ optical flow patches. Since the optical flow database was created from the Brown range image database by pairing range images with camera motions, spaces of small patches in optical flow may have same topological structures as that of small patches of range images (see the papers [2], [20]); moreover the authors of the paper [1] have found a similar topological structure for $3 \times 3$ optical flow patches.

In this paper, we analyze the structure of high-contrast regions of optical flow instead of looking at an optical flow as a whole, we are interested in the topology of the space of $n \times n$ high-contrast patches with sufficiently small $n$. 
We use the methods of the paper [7] to high-contrast optical flow patches, we study the topological features of spaces of $3 \times 3,5 \times 5$, and $7 \times 7$ patches of optical flow. In particular, we detect that there exist density subsets of $5 \times 5$ and $7 \times 7$ optical flow patches which are topologically equivalent to a circle (called the primary circle) and a three circles model, respectively. Adams, Atanasov, and Carlsson [1] have shown that $3 \times 3$ optical flow patches have the primary circle behavior. Furthermore, we show that there exists a two-dimensional subspace of $3 \times 3$ optical flow patches whose topology is that of the Klein bottle. As sizes of patches increase, the Klein bottle feature of the spaces gradually weakens. The results of this paper indicate that some results of topological analysis of natural images and range images can be extended to the field of image motion.

In Section 2 we survey persistent homology, and in Section 3 we describe our data sets selected from the optical flow database. We give results on core subsets in Section 4. We establish the relation between the spaces of optical flow patches and the space of two-variable polynomials in Section 5, present results on our data sets in Section 6, and summarize in Section 7.

\section{Persistent homology}

Given a point-cloud dataset $P$ sampled from an underlying space $X$, one wants to build a simplicial complex $K$ to approximate the topological structure of $X$. A natural approach is to express the data by a simplicial complex $K$, with the data points as vertices, and adding edges, triangles and high-dimensional cells according to proper rules. If $K$ is an accurate reliable topological representation of $X$, then one can obtain Betti numbers $\beta_{k}=\beta_{k}(X)$, by computing the numbers $\beta_{k}(K)([8])$, the $k$-th Betti number $\beta_{k}$ is the rank of the $k$-th homology group of $K$. A number of simplicial complexes can be built from a sampled point set of $X$. Due to the dimensional issue, in practice we usually apply lazy witness complexes defined in [8].

For a larger dataset $P$, we choose a subset $L \subset P$, called landmark points, as the only vertices. All points in $P$ help to serve as witnesses for the inclusion of high-dimensional simplices. The most common method for choosing landmark points is a greedy inductive selection process called the sequential maxmin. In the sequential maxmin, the first landmark is chosen randomly from $P$. Inductively, if $s_{1}, \ldots, s_{i-1}$ have been chosen, let $s_{i} \in P \backslash\left\{s_{1}, \ldots, s_{i-1}\right\}$ be the data point which maximizes the function $t \mapsto \min \left\{d\left(t, s_{1}\right), \ldots, d\left(t, s_{i-1}\right)\right\}$, where $d\left(t, s_{j}\right)$ is the distance between two points $t, s_{j}, j=1, \ldots, i-1$. Continue until the required number of landmark points have been chosen.

Fix a point cloud $P$, a landmark subset $L$, and a parameter $n \in \mathbb{N}$. If $n=0$, let $m(p)=0$ for all $p \in P$. If $n>0$, let $m(p)$ be the distance from $p$ to the $n$-th closest landmark point. The lazy witness complex $\operatorname{LW}_{n}(P, L, \varepsilon)$ 
is defined as follows: (i) the vertex set is $L$; (ii) for vertices $a$ and $b$, the edge $[a b]$ is in $\operatorname{LW}_{n}(P, L, \varepsilon)$ if there exists a witness point $p \in P$ such that $\max \{d(a, p), d(b, p)\} \leq \varepsilon+m(p)$; (iii) a high-dimensional simplex is in $\mathrm{LW}_{n}(P, L, \varepsilon)$ if all of its edges are in it.

The lazy witness complex depends on a parameter $n \in\{0,1,2\}$ which we choose to be $n=1$ : De Silva and Carlsson in [8] find $n=0$ to be generally less effective, while $n=2$ has the disadvantage of connecting every landmark point to at least one another at $\varepsilon=0$. For more information and examples about lazy witness complexes we refer to the papers [8], [3].
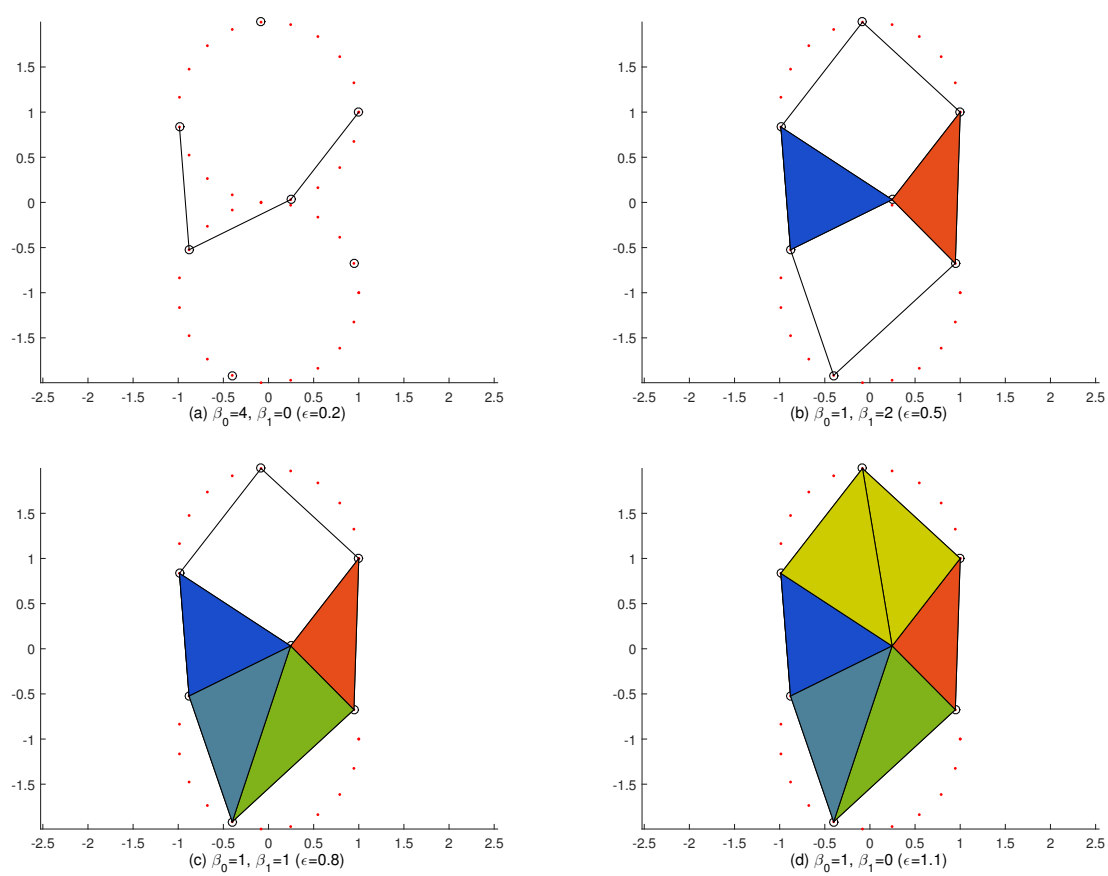

Figure 1. A sequence of lazy witness complexes for a point cloud data set sampled from the figure " 8 ". As $\varepsilon$ increases, holes appear and disappear.

Converting a point cloud data set into a lazy witness complex one selects a parameter $\varepsilon$. If $\varepsilon$ is small enough, then the complex will be a discrete set. If $\varepsilon$ is large enough, the complex will be a single high-dimensional simplex. Is there an optimal selection for $\varepsilon$ which best describes the topology of the data set? We consider the set of 40 points sampled from the figure "8" and a sequence of lazy witness complexes illustrated in Figure 1(a)-(d), here we take seven landmark points, $n=1$ and $\varepsilon=0.2,0.5,0.8,1.1$, respectively. The point set has Betti numbers $\beta_{0}=1$ and $\beta_{1}=2$. Can this be deduced? We observe that there is an inclusion of the current complex into the next complex (from (a) to (d)), since 
the current one corresponds to a smaller parameter value than the next one. In Figure 1, we note that there is no hole in Figure 1 (a), two new holes appear in Figure 1(b). As $\varepsilon$ increases, one hole vanishes, see Figure 1 (c), and the other hole disappears, see Figure $1(\mathrm{~d})$. We note that the figure " 8 " consists exactly of two holes, therefore Figure 1 (b) is the correct answer.

In spite of being computable and insightful, the homology of a complex associated to a point cloud at a specific $\varepsilon$ is not sufficient: there is no optimal value of $\varepsilon$. One needs means of declaring which holes are essential and which can be safely ignored. The standard topological constructions of homology and homotopy offer no such slack in their strident rigidity: a hole is a hole no matter how fragile or fine ([12]).

If we have only finite sampled points from an underlying space $X$, and no a priori information about the space $X$, it is very hard to measure a value of $\varepsilon$ that creates a simplicial complex whose homology group is isomorphic to that of $X$. In order to solve the problem, Edelsbrunner, Letscher, and Zomorodian introduced persistence in [9], which was refined by Zomorodian and Carlsson in [21]. For $\varepsilon \leq \varepsilon^{\prime}$, there exists a natural inclusion of simplicial complexes $\operatorname{LW}_{n}(P, L, \varepsilon) \hookrightarrow$ $\mathrm{LW}_{n}\left(P, L, \varepsilon^{\prime}\right)$, and because of the functoriality described above, there is a linear transformation $H_{k}\left(\mathrm{LW}_{n}(P, L, \varepsilon)\right) \rightarrow H_{k}\left(\mathrm{LW}_{n}\left(P, L, \varepsilon^{\prime}\right)\right)$ for each $k \geq 0$, where $H_{k}\left(\mathrm{LW}_{n}(P, L, \varepsilon)\right)$ is the $k$-th homology group of $\operatorname{LW}_{n}(P, L, \varepsilon)$. A directed system $H_{k}\left(\mathrm{LW}_{n}(P, L, \varepsilon)\right)$ is called a persistence vector space [9], for each persistence vector space there exists an invariant called a barcode, which is a finite collection of intervals. It is proved that barcodes are in bijective correspondence with isomorphism classes of directed systems of vector spaces ([21]). The barcodes are computable from sampled points using the PLEX software package developed by Adams and Tausz ([3]).

Intuitively, the intervals of a barcode mean the lifetimes of the topological properties. The left endpoint of an interval corresponds to the birth of a topological property, and the right endpoint corresponds to its death. Recall that the $k$-th Betti number of a complex, $\beta_{k}=\operatorname{rank} H_{k}$, is roughly equal to the number of $k$-dimensional holes, and $\beta_{k}$ may be calculated by the barcodes, that is, it is the number of intervals in the dimension $k$ plot that intersect the vertical line through $\varepsilon$ ( $\varepsilon$-values are on the horizontal axis). Long intervals mean holes persisting through a large range of values of the parameter $\varepsilon$ while short intervals correspond to holes filled in quickly. The interpretation by intuition is that long intervals correspond to real topological properties of the underlying space while short ones are topological noise coming from the point sampling. Figure 2 shows an example of barcode representations of the homology of the 40 points from the figure " 8 " (see the shape of red points in Figure 1(a)): for the case $\varepsilon=0.2$, the barcodes give $\beta_{0}=4, \beta_{1}=0$, which are Betti numbers of the lazy witness 
complex in Figure 1 (a); for the range of $\varepsilon \in[0.3,0.78]$, in particular, $\varepsilon=0.5$, the barcodes give $\beta_{0}=1, \beta_{1}=2$ and $\beta_{2}=0$, which are Betti numbers of the lazy witness complex in Figure 1(b); for the case $\varepsilon=0.8$, the barcodes show $\beta_{0}=1$, $\beta_{1}=1$, which are Betti numbers of the lazy witness complex in Figure $1(\mathrm{c})$. In Figure 2 there are one long line in dimension 0, two long lines in dimension 1 (from $\varepsilon_{0}=0.3$ to $\varepsilon_{1}=0.78$ ) and no line in dimension 2, which responds to the fact that " 8 " has one connected component and two holes in dimension one.

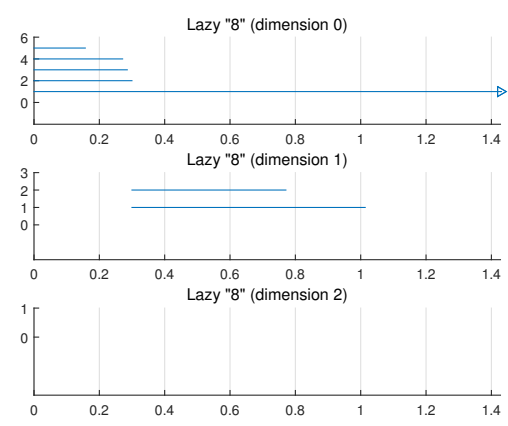

Figure 2. The barcodes for the sequence of lazy witness complexes $\mathrm{LW}_{1}(P, L, \varepsilon), 0 \leq \varepsilon \leq 1.4$, built in Figure 1 .

\section{Spaces of optical flow patches}

Our spaces are constructed from the Roth and Black optical flow database [16], which is available at http://www.visinf.tu-darmstadt.de/vi_ research/ vi_datasets/vi_flowstats.en.jsp. Figure 3 has two samples.

We randomly select data sets of high-contrast $3 \times 3,5 \times 5$, and $7 \times 7$ patches from the optical flow database. Our main spaces $X_{3}, X_{5}$, and $X_{7}$ are sets of $3 \times 3,5 \times 5$, and $7 \times 7$ patches of high-contrast created by the following six steps, the steps here are analogous to [15], [7], [2]. The spaces $X_{3}$ and $X_{5}$ have about 220000 points, but $X_{7}$ has only about 114500 points.

Step 1. For each flow field sequence in the database, we use the second optical flow frame of a sequence, and randomly select $1500 m \times m(m=3,5)$ patches, and $8007 \times 7$ patches respectively from it. One $3 \times 3$ patch is arranged as

$$
\left(\begin{array}{lll}
\left(u_{1}, v_{1}\right) & \left(u_{4}, v_{4}\right) & \left(u_{7}, v_{7}\right) \\
\left(u_{2}, v_{2}\right) & \left(u_{5}, v_{5}\right) & \left(u_{8}, v_{8}\right) \\
\left(u_{3}, v_{3}\right) & \left(u_{6}, v_{6}\right) & \left(u_{9}, v_{9}\right)
\end{array}\right),
$$

where $u$ indicates the optical flow in the horizontal direction and $v$ indicates the vertical direction. We treat each $3 \times 3$ patch as a vector $\mathbf{x}=\left(u_{1}, \ldots, u_{9}, v_{1}, \ldots, v_{9}\right)$ in $\mathbb{R}^{18}$. 


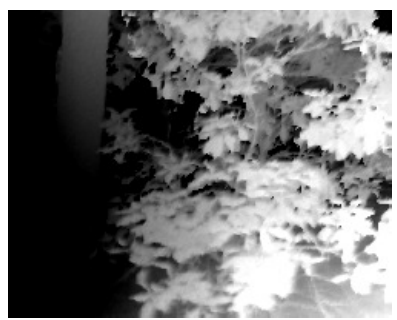

(a)

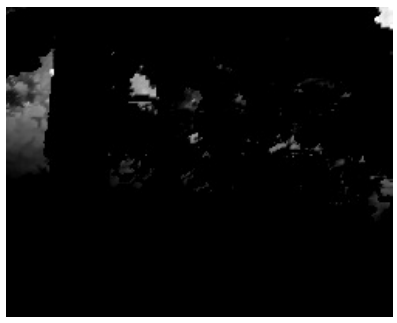

(c)

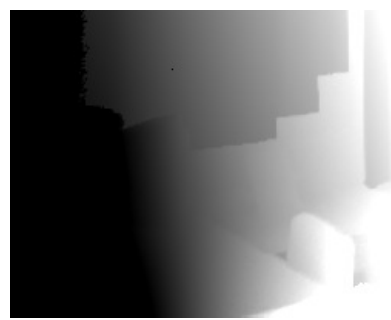

(b)

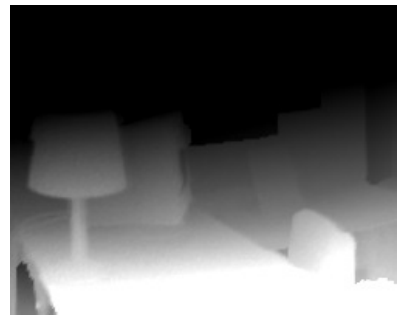

(d)

Figure 3. Two samples from the optical flow database. Horizontal motions are on the top and vertical motions are on the bottom.

Step 2. We calculate the $D$-norm: $\|\mathbf{x}\|_{D}$ for each vector $\mathbf{x}$, which is a measure of contrast of an optical flow patch. Two pairs of coordinates $\left(u_{i}, v_{i}\right),\left(u_{j}, v_{j}\right)$ of $\mathrm{x}$ are adjacent, denoted by $i \sim j$, if the corresponding pixels in the $m \times m$ patch are neighbors. We calculate the $D$-norm for a vector $\mathbf{x}$ by the formula:

$$
\|\mathbf{x}\|_{D}=\sqrt{\sum_{i \sim j}\left\|\left(u_{i}, v_{i}\right)-\left(u_{j}, v_{j}\right)\right\|^{2}} .
$$

Step 3. We pick the patches that have a $D$-norm in the top $T$ percent of each sequence. We set $T=20 \%$, as done in [15], [7], [2].

Step 4. For $u$ components, subtract an average of $u$ coordinates, and for $v$ components, subtract an average of $v$ coordinates, this produces a new $2 m^{2}$ dimensional vector.

Step 5. We transfer the spaces into a unit sphere by dividing each vector with its Euclidean norm, which is not zero because the patches are high-contrast. We do not transform to the DCT basis for convenience.

Step 6. For the convenience of calculations, we randomly take 50000 of the above patches in the top $20 \%$, and these sets are subspaces of $X_{3}, X_{5}$, and $X_{7}$, denoted by $X S_{3}, X S_{5}$, and $X S_{7}$ respectively. 


\section{Results for $X S_{m}(k, p)$}

The facts about $3 \times 3,5 \times 5$, and $7 \times 7$ range image patches having core subsets with topological feature of a circle were established by Xia in [20] and Adams and Carlsson in [2]. We use core subsets to explore a circular feature in spaces $X S_{3}, X S_{5}$, and $X S_{7}$. We introduce the concept of core subsets of a space $Y$. For $y \in Y$ and $k>0$, set $\rho_{k}(y)=\left|y-y_{k}\right|$, where $y_{k}$ is the $k$-th nearest neighbour of $y$. For a fixed $k$, we order points of $Y$ by descending density, we choose the points indicated by $Y(k, p)$ whose densities are in the top $p$ percents. The core subset $Y(k, p)$ may have more important topological information than that of all the points of $Y$.

Persistent homology is a method for computing topological characteristics of a space by using only finite samples of the space. We use lazy witness complexes and the software package PLEX to compute persistent homology, for more details, we refer to [7], [8], [3], [21], [6]. The result of a sequence of complexes (or a point set) computed by the software package PLEX is represented by a figure and often known as a PLEX result.

We consider the core subsets $X S_{3}(100,30), X S_{5}(100,30)$, and $X S_{7}(100,30)$, and compute their barcodes, sample Betti barcode plots for these core subsets are displayed in Figures 4, 5 and 6, respectively. There exist a long Betti interval $_{0}$ and a long Betti interval in the plots, which means $\beta_{0}=1, \beta_{1}=1$, that is, with the topology of a circle. Choosing different landmark points (from 30 to 60 ), we do three hundred trials on $X S_{3}(100,30), X S_{5}(100,30)$, and $X S_{7}(100,30)$, in each trial result, the circular feature $\beta_{0}=\beta_{1}=1$ is found in a long interval, and other Betti plot intervals are very small, so the results are stable. For various sizes of optical flow patches, there exist different core subsets of $X S_{3}, X S_{5}$, and $X S_{7}$ with appropriate values of parameters $k$ and $p$, which have the topology of a circle. We ran about five hundred experiments and discovered that the core subsets with the following parameter values have the homology of a circle and the results are sturdy: $m=3, k=200,300, p=30 ; m=5, k=200,300, p=30$; $m=7, k=200,300, p=30$.

\section{A space of two-variable polynomials and the Klein bottle}

The Klein bottle can be represented by an identification space of a square, see Figure 7 . In the process of pasting a square, three circles are formed, one is the primary circle (corresponding to $S_{\text {lin }}$ ) formed by horizontal segments (black lines), the other two circles (corresponding to $S_{\mathrm{v}}$ and $S_{\mathrm{h}}$ ) come from the vertical segments (red and blue lines) respectively, which is called the three circles model (Figure 8), denoted as $C_{3}$.

In order to identify the topological characteristics of subspaces of $X_{3}, X_{5}$, and $X_{7}$, we construct another theoretical model of the Klein bottle. We consider 


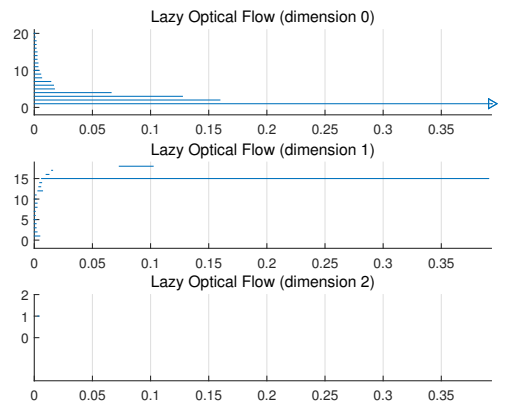

Figure 4. Barcodes for $X S_{3}(100,30)$.

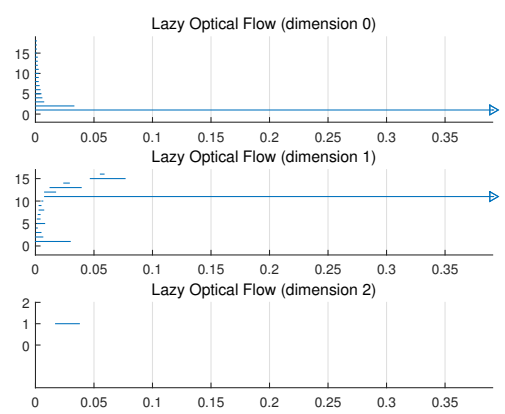

Figure 6. Barcodes for $X S_{7}(100,30)$.

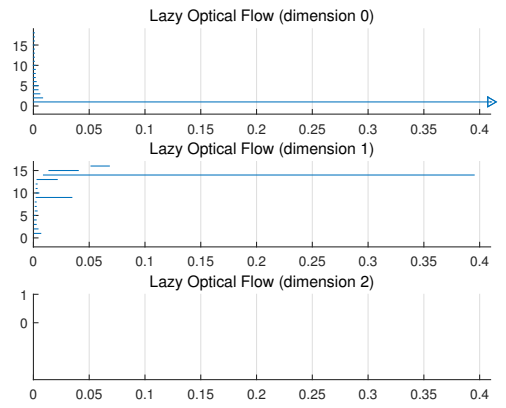

Figure 5. Barcodes for $X S_{5}(100,30)$.

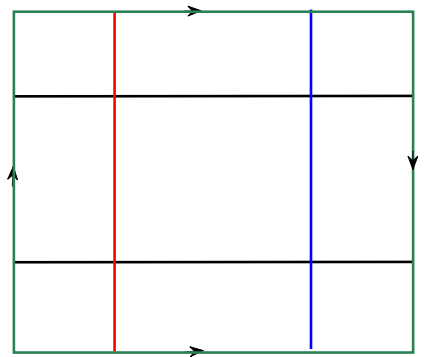

FiguRE 7. Denotation of the Klein bottle as an identification space.

$m \times m$ optical flow patches as gained by sampling a smooth real-valued function on the $x y$-plane at $m \times m$ grid points. We now study the space $\mathcal{F}$, consisting of all functions of the form $a_{2}\left(a_{1} x+b_{1} y\right)^{2}+b_{2}\left(a_{1} x+b_{1} y\right)$, where $a_{1}, b_{1}, a_{2}, b_{2}$ are real numbers, and $\left(a_{1}, b_{1}\right) \in S^{1},\left(a_{2}, b_{2}\right) \in S^{1}$, where $S^{1}$ stands for the unit circle in the plane.

We define the mapping $g: S^{1} \times S^{1} \rightarrow \mathcal{F}$ by $\left(a_{1}, b_{1}, a_{2}, b_{2}\right) \mapsto a_{2}\left(a_{1} x+b_{1} y\right)^{2}+$ $b_{2}\left(a_{1} x+b_{1} y\right)([7])$. It is easy to see that the map $g$ is onto, but not one-to-one, since the points $\left(a_{1}, b_{1}, a_{2}, b_{2}\right)$ and $\left(-a_{1},-b_{1}, a_{2},-b_{2}\right)$ are mapped to the same function, that is, $\left(a_{1}, b_{1}, a_{2}, b_{2}\right) \sim\left(-a_{1},-b_{1}, a_{2},-b_{2}\right)$ is an equivalence relation. When $\left(a_{1}, b_{1}, a_{2}, b_{2}\right)$ is represented by $(\cos \alpha, \sin \alpha, \cos \beta, \sin \beta)$, both $\alpha$ and $\beta$ vary in $[0,2 \pi]$, then the relation can be rewritten as $(\alpha, \beta) \sim(\pi+\alpha, 2 \pi-\beta)$. The space $\mathcal{F}=\operatorname{im}(g)$ is homeomorphic to $S^{1} \times S^{1} /(\alpha, \beta) \sim(\pi+\alpha, 2 \pi-\beta)$, since no other identifications are formed by $g$.

The torus has a similar representation to that of the Klein bottle as a square with pasted opposite edges, but pasting them with reverse orientation. The result 


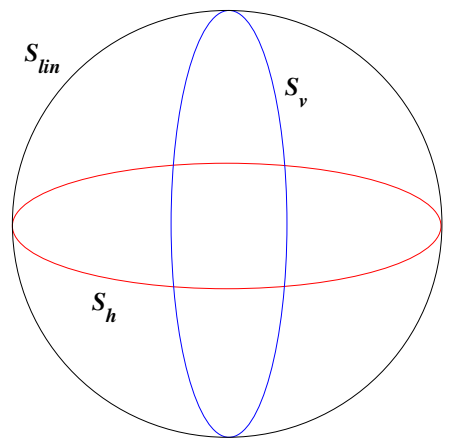

Figure 8. The three circles model.

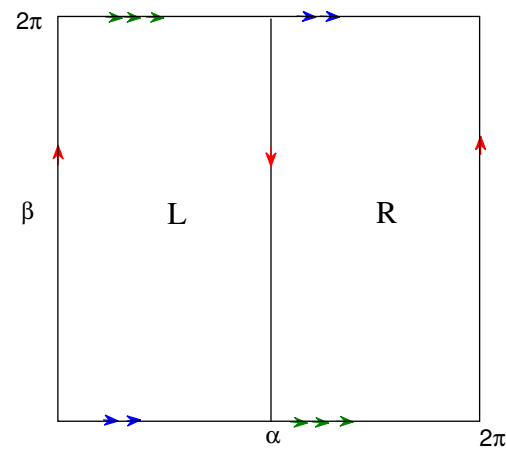

Figure 9. The Klein bottle, the image of the map $g$.

of the map $g$ on the torus is displayed in Figure 9. Each half is an indication of the Klein bottle, thus the image of $g$ is homeomorphic to the Klein bottle and so is $\mathcal{F}$ (see [7]).

The model $C_{3}$ is involved in the space $\mathcal{F}$. The primary circle of $C_{3}$ is the subspace obtained by setting $\left(a_{2}, b_{2}\right)=(0,1)$ and $\left(a_{1}, b_{1}\right)$ running on $S^{1}$, while the other circles are obtained by setting $a_{1}=1, b_{1}=0$ and $a_{1}=0, b_{1}=1$, respectively.

Define a mapping $h_{3}: \mathcal{F} \rightarrow S^{17}$ evaluating the function at each point of the plane grid $G_{3}=\{-2,-1,0,1,2,3\} \times\{-1,0,1\}$, subtracting the mean and normalizing. Define a mapping $h_{5}: \mathcal{F} \rightarrow S^{49}$ evaluating the function at each point of the plane grid $G_{5}=\{-4,-3,-2,-1,0,1,2,3,4,5\} \times\{-2,-1,0,1,2\}$, subtracting the mean and normalizing. Define a mapping $h_{7}: \mathcal{F} \rightarrow S^{97}$ evaluating the polynomial at each point of the plane grid $G_{7}=\{-6,-5,-4,-3,-2,-1,0,1,2$, $3,4,5,6,7\} \times\{-3,-2,-1,0,1,2,3\}$, subtracting the mean and normalizing.

Proposition 5.1. Each $h_{m}, m=3,5,7$, is one-to-one.

The proof is similar to the proof in [7].

As a continuous one-to-one map on a compact space is a homeomorphism onto its image, the image $\operatorname{im}\left(h_{m} \mid \mathcal{F}\right), m=3,5,7$, is homeomorphic to the Klein bottle, by Proposition 5.1.

In order to insert the space $C_{3}$ into the unit sphere $S^{17}$, we randomly select 400 points $\left\{\left(x_{1}, y_{1}\right), \ldots,\left(x_{400}, y_{400}\right)\right\}$ from $S^{1}$, then set $a_{2}=0, b_{2}=1 ; a_{1}=1$, $b_{1}=0 ; a_{1}=0, b_{1}=1$, respectively, for each of the 400 points, we compute its image under the map $h_{3} \circ \mathrm{g}$, denoting the set of all images as $C_{3}^{3}(400)$. If we compute the images of the 400 points under the mappings $h_{5} \circ g$ and $h_{7} \circ g$, respectively, we get $C_{3}^{5}(400)$ and $C_{3}^{7}(400)$, respectively. Figure 10 displays the PLEX result for the spaces $C_{3}^{3}(400)$, it gives the topology of $C_{3}$, i.e. $\beta_{0}=1$, 
$\beta_{1}=5$, hence we obtain an appropriate approach of $C_{3}$ in $S^{17}$. We have the same results for the spaces $C_{3}^{5}(400)$ and $C_{3}^{7}(400)$ (see Figures 11 and 12).

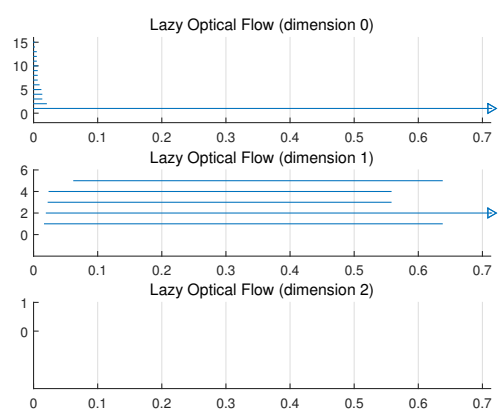

Figure 10. Barcodes for $C_{3}^{3}(400)$.
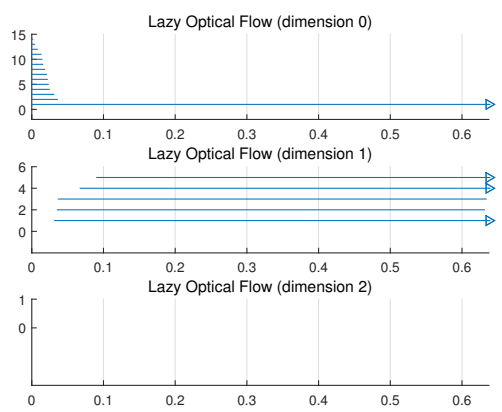

Figure 12. Barcodes for $C_{3}^{7}(400)$.
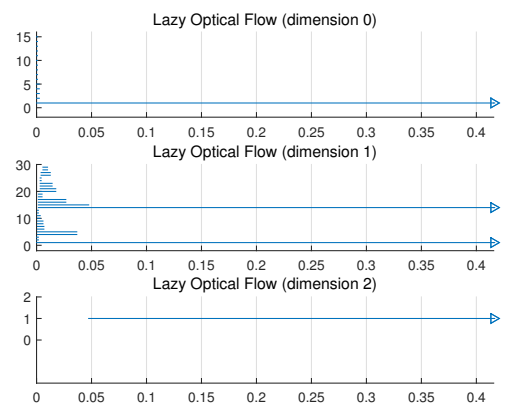

Figure 14. Barcodes for $K^{5}(200)$.

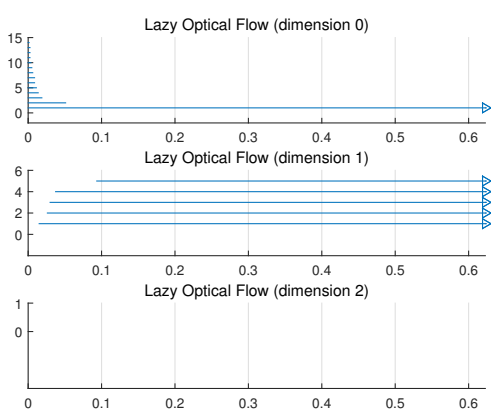

Figure 11. Barcodes for $C_{3}^{5}(400)$.
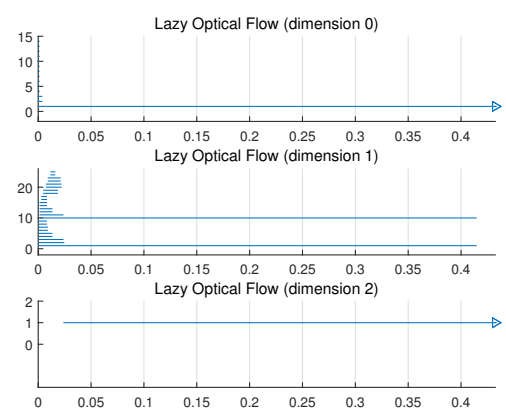

Figure 13. Barcodes for $K^{3}(200)$.
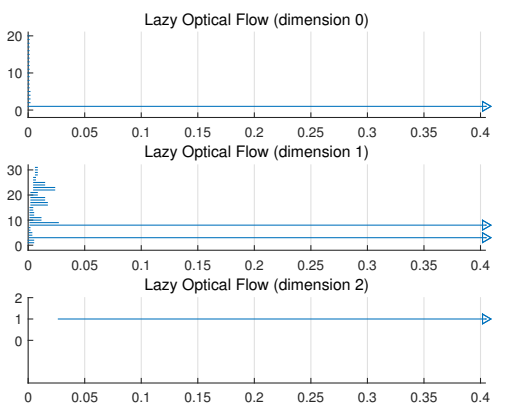

Figure 15. Barcodes for $K^{7}(200)$.

In order to insert the Klein bottle into a unit sphere, firstly, we uniformly pick 200 points $\left(\left\{x_{1}, \ldots, x_{200}\right\}\right)$ on the unite circle, all possible tuples $\left(x_{i}, x_{j}\right)$ form a point set on the torus $S^{1} \times S^{1}$. Second, we map each of the 40000 points 
into $S^{17}, S^{49}$ and $S^{97}$ by mappings $h_{3} \circ g, h_{5} \circ g$, and $h_{7} \circ g$, respectively, the image of each mapping is represented by $K^{3}(200), K^{5}(200)$, and $K^{7}(200)$, respectively. Figure 13 shows the PLEX result for the spaces $K^{3}(200)$, that gives $\beta_{0}=1$, $\beta_{1}=2$ and $\beta_{2}=1$, which are the mod 2 Betti numbers of the Klein bottle. Hence, $K^{3}(200)$ is an appropriate approach of the Klein bottle in $S^{17}$. We have similar results for the spaces $K^{5}(200)$ and $K^{7}(200)$ (see Figures 14 and 15).

\section{Results for $X_{3}, X_{5}$ and $X_{7}$}

In order to detect subspaces of $X_{3}$ whose topology is that of $C_{3}$, for any point $p \in C_{3}^{3}(400)$, we collect the points of $X_{3}$ that are closest points to $p$ (in Euclidean distance), the subspace of $X_{3}$ is written as $C C_{3}^{3}(400)$. One sample PLEX Betti barcode plot for $C C_{3}^{3}(400)$ is given in Figure 16, which indicates that $C C_{3}^{3}(400)$ has Betti numbers $\beta_{0}=1$ and $\beta_{1}=5$, that is, it has the topology of $C_{3}$. Similarly, we get the same results for the spaces $C C_{3}^{5}(400)$ and $C C_{3}^{7}(400)$ (Figures 17 and 18).

REMARK 6.1. The Betti barcode result for the space $C C_{3}^{5}(400)$ is not very stable, sometimes it does not have the homology of $C_{3}$. We ran 200 experiments on $C C_{3}^{5}(400)$, there are only 118 experiments whose PLEX barcodes have $\beta_{0}=1$, $\beta_{1}=5$.

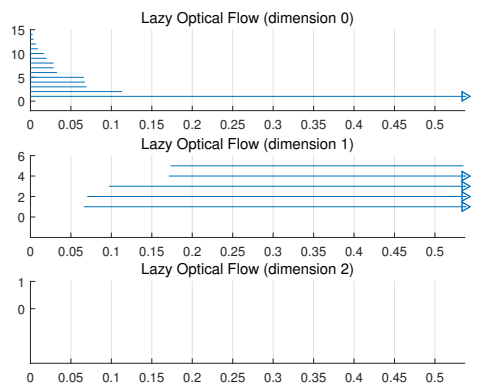

Figure 16. Barcodes for $C C_{3}^{3}(400)$.

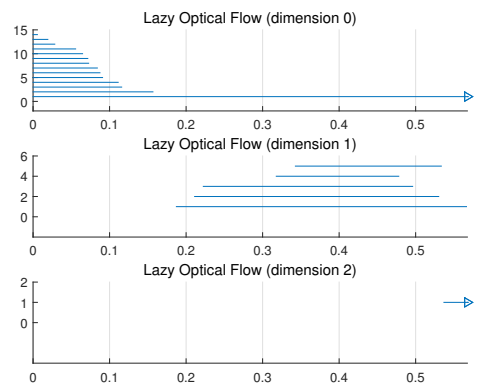

Figure 17. Barcodes for $C C_{3}^{5}(400)$.

We have shown in the previous section that $S^{17}, S^{49}$ and $S^{97}$ have subspaces $K^{3}(200), K^{5}(200)$ and $K^{7}(200)$, respectively, whose homology is that of the Klein bottle, subspaces of $X_{3}, X_{5}$, and $X_{7}$ can be constructed by using them. Next we describe the method how to get the subspaces of $X_{3}, X_{5}$, and $X_{7}$.

For each point $p$ of $K^{3}(200)$ we compute the Euclidean distance from $p$ to every point of $X_{3}$, then pick up $t$ closest points to the point $p$. The subspace of $X_{3}$ is constructed by selecting all $t$ closest points to any $p \in K^{3}(200)$, denoted by $\operatorname{Kopt}^{3}(200, t)$. The subspaces $\operatorname{Kopt}^{5}(200, t)$ and $\operatorname{Kopt}^{7}(200, t)$ of $X_{5}$ and $X_{7}$, respectively, are obtained by the same way. 


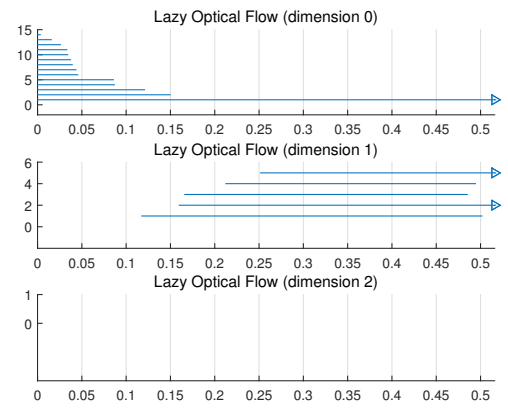

Figure 18. Barcodes for $C C_{3}^{7}(400)$.
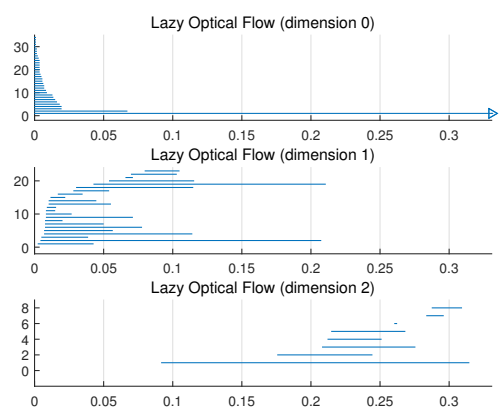

Figure 20. Barcodes for $\operatorname{Kopt}^{5}(200,10)$.

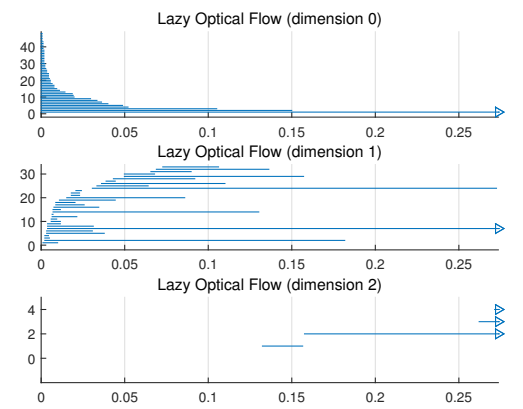

Figure 19. Barcodes for $\operatorname{Kopt}^{3}(200,10)$.

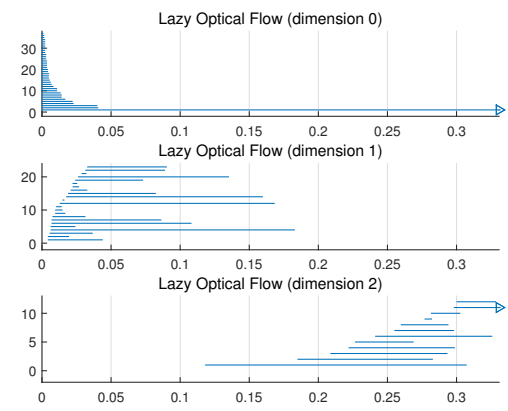

Figure 21. Barcodes for $\operatorname{Kopt}^{5}(200,10)$.

In order to find a subspace of $X_{3}$ with the homology of the Klein bottle, we utilize the subspace $\operatorname{Kopt}^{3}(200,10)$. Figure 19 shows that $\operatorname{Kopt}^{3}(200,10)$ has the homology of the Klein bottle with the parameter values from 0.18 to 0.26. In experimental results, some ranges of parameter values with the homology of the Klein bottle are very small.

Figures 20-22 display three PLEX results for $\operatorname{Kopt}^{5}(200,10)$. In Figure 20, the PLEX barcodes indicate $\beta_{0}=1, \beta_{1}=2$, and $\beta_{2}=1$ from 0.116 to 0.175 . In Figure 21, the PLEX barcodes give $\beta_{0}=1, \beta_{1}=2$, and $\beta_{2}=1$ in a very small range from 0.16 to 0.168 . But in Figure 22, the PLEX barcodes show no Klein bottle feature in $\operatorname{Kopt}^{5}(200,10)$. We ran 200 trials on $\operatorname{Kopt}^{5}(200, t)$ for $t=10,11,12$, there are only 89 experiments whose PLEX barcodes give $\beta_{0}=1$, $\beta_{1}=2$, and $\beta_{2}=1$, and some barcode intervals with the homology of the Klein bottle are very short, the others do not have the homology of the Klein bottle.

Figures 23-25 show three PLEX results for $\operatorname{Kopt}^{7}(200,10)$. In Figure 23, the PLEX barcodes show $\beta_{0}=1, \beta_{1}=2$, and $\beta_{2}=1$ from 0.154 to 0.197. In Figure 24 , the PLEX barcodes show $\beta_{0}=1, \beta_{1}=2$, and $\beta_{2}=1$ in a very small 

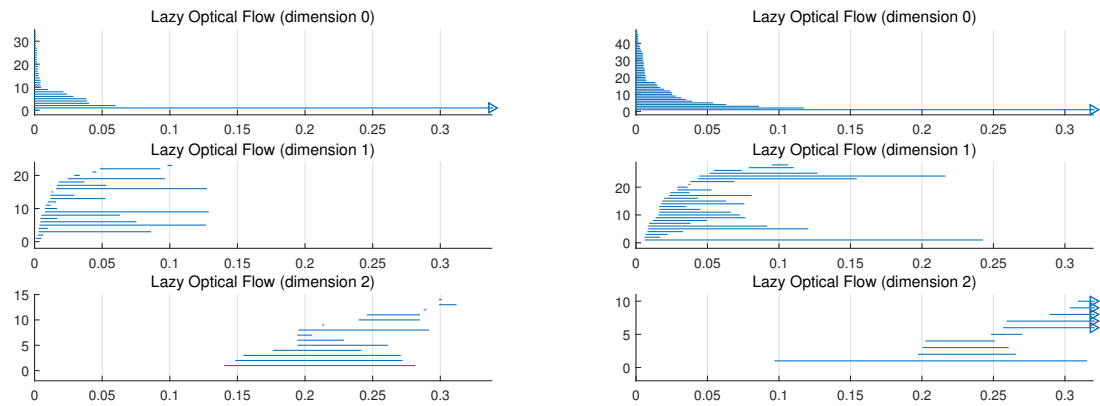

Figure 22. Barcodes for $\operatorname{Kopt}^{5}(200,10)$.

Figure 23. Barcodes for $\operatorname{Kopt}^{7}(200,10)$.
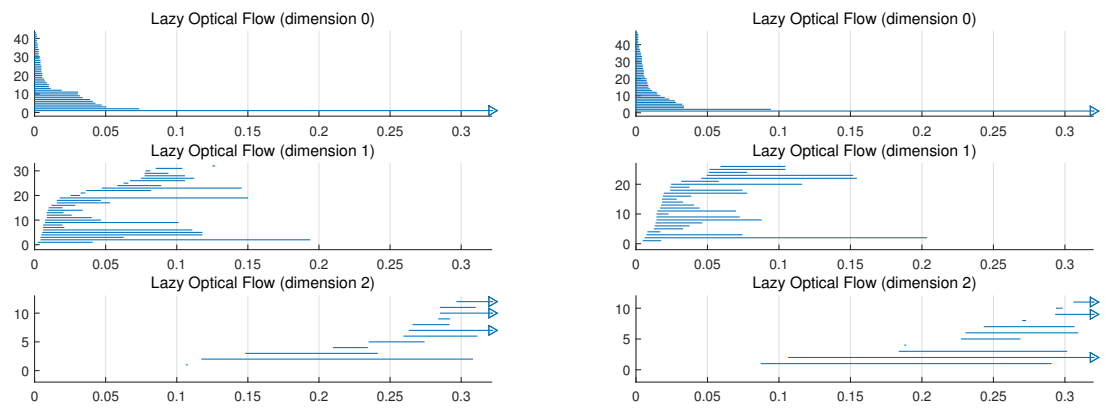

Figure 24. Barcodes for $\operatorname{Kopt}^{7}(200,10)$.

Figure 25. Barcodes for $\operatorname{Kopt}^{7}(200,10)$.

range from 0.145 to 0.149 . The PLEX barcodes in Figure 25 do not have the Klein bottle feature for $\operatorname{Kopt}^{7}(200,10)$. We ran 200 trials on $\operatorname{Kopt}^{7}(200, t)$ for $t=10,11,12$, there are only 67 trials whose PLEX barcodes give $\beta_{0}=1, \beta_{1}=2$, and $\beta_{2}=1$, and some barcode intervals with the homology of the Klein bottle are very small, the others do not have the homology of the Klein bottle.

Figures 20-22 indicate that the subspace $\operatorname{Kopt}^{5}(200,10)$ of $X_{5}$ undergoes a topological change. There is a similar result for the subspace $\operatorname{Kopt}^{7}(200,10)$ of $X_{7}$.

REMARK 6.2. We also study $\operatorname{Kopt}^{7}(w, t)$ for $w=180,220,260,280$, and $t=10,11,12$, we ran many experiments on them, which give similar results as for $\operatorname{Kopt}^{7}(200,10)$, thus we may conclude that the Klein bottle characteristics of the spaces $X_{3}, X_{5}$, and $X_{7}$ gradually disappears as the size of patches increases.

\section{Conclusions}

In this paper we use techniques of persistent homology to study topological qualitative analysis of spaces of small optical flow patches. We show that the 
spaces of high-contrast $3 \times 3,5 \times 5$, and $7 \times 7$ patches have core subsets modeled as the primary circle, and each of the spaces $X_{3}, X_{5}$, and $X_{7}$ has subspaces with topology of $C_{3}$. Using the relation between the spaces of optical flow patches and the space of two variables polynomials, it is proven that there exist subspaces of $X_{3}$, whose homology is that of a Klein bottle. Using the same methods to the various sizes optical flow patches, we find many similar properties for different sizes optical flow patches, but they also have their own features. The Klein bottle's feature of $X_{3}, X_{5}$, and $X_{7}$ depends on the size of optical flow patches, the the larger patches of spaces, the less of Klein bottle's feature they have, the results show that it is necessary to study different size patches in optical flow. Our results extend research on the topological analysis in images to the field of image motion.

Acknowledgements. The authors are very grateful to the reviewers for valuable comments and corrections.

\section{REFERENCES}

[1] H. Adams, A. Atanasov and G. Carlsson, Nudged elastic band in topological data analysis, Topol. Methods Nonlinear Anal. 45 (2015), 247-272.

[2] H. Adams And G. CARlsson, On the nonlinear statistics of range image patches, SIAM J. Imag. Sci. 2 (2009), 110-117.

[3] H. Adams And A. TAusz, Javaplex tutorial, http://javaplex.googlecode.com/svn/trunk /reports/javaplex_tutorial/javaplex_tutorial.pdf.

[4] S. Baker, D. Scharstein, J.P. Lewis, S. Roth, M. J. Black and R. Szeliski, A database and evaluation methodology for optical flow, Internat. J. Comput. Vision 92 (2011), $1-31$.

[5] J.L. Barron, D.J. Fleet and S.S. Beauchemin, Performance of optical flow techniques, Internat. J. Comput. Vision 12 (1994), 43-77.

[6] G. Carlsson, Topology and data, Bull. Amer. Math. Soc. (N.S.) 46 (2009), 255-308.

[7] G. Carlsson, T. Ishrhanov, V. De Silva and A. Zomorodian, On the local behavior of spaces of natural images, Internat. J. Comput. Vision, 76 (2008), 1-12.

[8] V. de Silva and G. Carlsson, Topological estimation using witness complexes, Proc. Sympos. Point-Based Graphics (2004), 157-166.

[9] H. Edelsbrunner, D. Letscher and A. Zomorodian, Topological persistence and simplification, Discrete Comput. Geom. 28 (2002), 511-533.

[10] D.J. FIELD, Relations between the statistics of natural images and the response properties of cortical cells, J. Opt. Soc. Amer. 4 (1987), 2379-2394.

[11] A. Geiger, P. Lenz and R. Untasun, Are we ready for autonomous driving? the kitti vision benchmark suite, CVPR (2012), 3354-3361.

[12] R. Ghrist, Barcodes: The persistent topology of data, Bull. Amer. Math. Soc. 45 (2008), 61-75.

[13] J.J. Gibson, The Perception of the Visual World, Riverside Press, Cambridge, 1950.

[14] K. JiA, X. WANG AND X. TANG, Optical flow estimation using learned sparse model, 2011 IEEE International Conference on Computer Vision, November (2011), 2391-2398.

[15] A.B. Lee, K.S. Pedersen and D. Mumford, The non-linear statistics of high-contrast patches in natural images, Internat. J. Comput. Vision 54 (2003), 83-103. 
[16] S. Roth, and M. J. Black, On the spatial statistics of optical flow, Internat. J. Comput. Vision 74 (2007), 33-50.

[17] D. Sun, S. Roth And M. J. Black, A quantitative analysis of current practices in optical flow estimation and the principles behind Them, Internat. J. Comput. Vision 106 (2014), 115-137.

[18] J.H. VAN HATEREN, Theoretical predictions of spatiotemporal receptive fields of fly LMCs, and experimental validation, J. Comput. Physiology A 171 (1992), 157-170.

[19] D.H. Warren and E.R. Strelow, Electronic Spatial Sensing for the Blind: Contributions from Perception, Springer, 1985.

[20] S. XIA, On the local behavior of spaces of range image patches, to appear.

[21] A. Zomorodian and G. Carlsson, Computing persistent homology, Discr. Comput. Geom. 33 (2005), 249-274.

Manuscript received February 23, 2016 accepted May 8, 2016

ShengXiang Xia And Yanmin Yin

College of Science

Shandong Jianzhu University

Jinan 250101, P.R. CHINA

E-mail address: xias@sdjzu.edu.cn, yym@sdjzu.edu.cn 